\title{
lonization effect in the atmosphere during several Halloween GLE events in October-November 2003
}

\author{
Alexander Mishev* \\ Space Climate Research Unit, University of Oulu, Finland; Sodankylä Geophysical Observatory, \\ University of Oulu, Finland. \\ E-mail: alexander.mishev@oulu.fi
}

\section{Peter I. Y. Velinov}

Institute for Space Research and Technology, Bulgarian Academy of Sciences, Sofia, Bulgaria

E-mail: pvelinov@bas.bg

Galactic cosmic rays are the main source of ionization in the Earth's stratosphere and troposphere. However, in some cases, specifically during solar maximum periods, eruptive solar processes, such as solar flares and coronal mass ejections lead to an acceleration of high-energy solar energetic particles (SEPs). The energy of SEPs is usually tens of MeV/nucleon, occasionally exceeding $100 \mathrm{MeV} /$ nucleon. Rarely, in some cases, it reaches about several GeV/nucleon. While the $\mathrm{MeV}$ particles can be absorbed in the atmosphere, those with energy about $\mathrm{GeV} / \mathrm{n}$ initiate an atmospheric cascade similarly to the galactic cosmic rays. This special class of events, called ground level enhancement can drastically enhance the atmospheric ionization. The induced by primary cosmic ray particles ionization is important for various processes related to the physics and chemistry of the atmosphere and minor constituents. During the solar cycle 23 were observed several strong ground level enhancements. Here, we study the ionization effect during the sequence of three ground level enhancements of October-November 2003, the so-called Halloween events. We apply a full Monte Carlo 3-D model for computation of the cosmic ray induced ionization as a function of the altitude above sea level and derive the corresponding ionization effect.

36th International Cosmic Ray Conference - ICRC 2019-

24 July-1 August, 2019

Madison, United States

\footnotetext{
${ }^{*}$ Speaker.
} 


\section{Introduction}

High-energy particles of galactic and/or solar origin are the main source of ionization in the low and middle atmosphere [1]. A systematic study of the impact ionization due to precipitating high-energy particles is very important in order to clarify their influence on different atmospheric processes, global electric circuit and atmospheric physics and chemistry, specifically of minor components $[2,3,4]$.

While in the upper atmosphere, the ion production is dominated by the contribution of solar $\mathrm{UV}$ and X-rays, below $100 \mathrm{~km}$ the atmospheric ionization is governed by relativistic electrons from radiation belts, precipitating electrons, auroral electrons, solar energetic particles (SEPs), galactic cosmic rays (GCRs). In this work we focus on ion production in the troposphere and stratosphere, therefore due to high-energy protons of galactic and/or solar origin.

The main atmospheric ionization in the tropospheric and stratospheric region is due to GCRs. When GCRs enter in the atmosphere, after interaction with air molecules, induce a complicated nuclear-electromagnetic-lepton cascade, eventually leading to an ionization of the ambient air [1]. The maximum of ion production is observed at altitude of about $12-15 \mathrm{~km}$ above sea level (a.s.1.), known as Regener-Pfotzer maximum [5, 6]. Occasionally, after strong solar eruptions, SEPs are accelerated to high-energies, which allow to produce an atmospheric cascade similarly to GCRs, events known as ground level enhancements (GLEs) [7, 8]. Therefore, strong SEP events can significantly enhance the ion production in the atmosphere, specifically over the polar and subpolar region $[9,10,11,12]$. Nowadays, recently developed models based on a full Monte Carlo simulation of CR propagation and interaction in the atmosphere, allow one to assess realistically the atmospheric ionization due to precipitating particles [13, 14, 15, 16].

During solar cycle 23 were observed 16 GLEs. A sequence of three GLEs was observed in the late October - early November 2003 by the global neutron monitor (NM) network (Fig.1). In addition, the period was characterized by a significant cosmic ray variability, namely a strong Forbush decrease was recorded, which is explicitly considered in this study. Using precise information of GLE particles spectra $[17,18]$ derived using NM data and a convenient model, we computed the ion production rate and the corresponding ionization effect during late October - early November 2003.

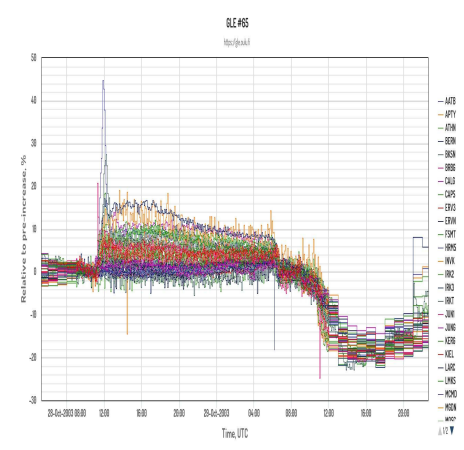

(a) GLE 65 on 28 October 2003.

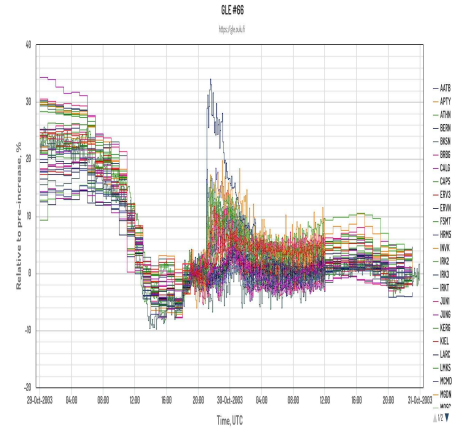

(b) GLE 66 on 29 October 2003.

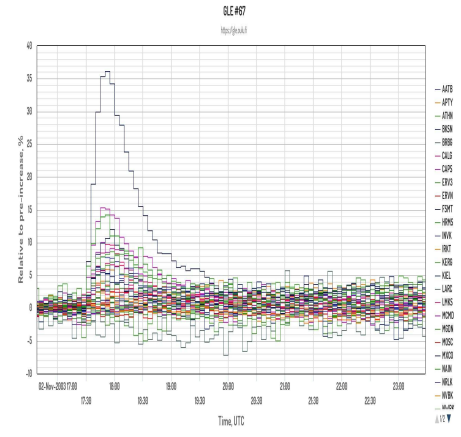

(c) GLE 67 on 2 Nov. 2003.

Figure 1: NM count rate variation during the sequence of three Halloween GLEs. 


\section{Used model for computation of ion production rate in the atmosphere}

Here we use model similar to [15], the full description is given elsewhere [16, 19]. The ion production rate is computed using the expression:

$$
q\left(h, \lambda_{m}\right)=\frac{1}{E_{\text {ion }}} \sum_{i} \int_{E_{\text {cut }}\left(R_{c}\right)}^{\infty} \int_{\Omega} D_{i}(E) \frac{\partial E(h, E)}{\partial h} \rho(h) d E d \Omega
$$

where $\partial E$ is the deposited energy in an atmospheric layer $\partial h, h$ is the air overburden above a given altitude in the atmosphere expressed in $\mathrm{g} / \mathrm{cm}^{2}$ subsequently converted to altitude a.s.1., $D_{i}(E)$ is the differential cosmic ray spectrum for a given component $i$ (proton, $\alpha$-particle, heavier nuclei), $\rho$ is the atmospheric density in $\mathrm{g} . \mathrm{cm}^{-3}, E$ is the initial kinetic energy of the incoming primary nuclei on the top of the atmosphere, $\Omega$ is a solid angle and $E_{i o n}=35 \mathrm{eV}$ is the energy necessary for creation of an ion pair in air [20]. The integration is over the kinetic energy above $E_{\text {cut }}\left(R_{c}\right)$, which is defined by the local rigidity cut-off $R_{c}$ for a nuclei of type $i$ at a given geographic location by the expression:

$$
E_{\text {cut }, i}=\sqrt{\left(\frac{Z_{i}}{A_{i}}\right)^{2} R_{c}^{2}+E_{0}^{2}}-E_{0}
$$

where $E_{0}=0.938 \mathrm{GeV} / \mathrm{n}$ is the proton's rest mass.

The ion production in the atmosphere during GLEs is a superposition of the contribution of GCRs and SEPs [21]. For the GCRs we assume the force field model [22, 23, 24], accordingly for SEPs spectra in equation (2.1), we consider derived spectra on the basis of NM data analysis similarly to $[25,26,27]$.

In this study, the propagation and interaction of high-energy protons with the atmosphere is simulated with the PLANETOCOSMICS code [14] assuming a realistic atmospheric model NRLMSISE 00 and considering seasonal influence [28, 29]. The model was recently applied for computation of ion production rate $[30,31,32]$ and the corresponding ionization effect during GLE events $[33,34,35,36]$.

\section{Ion production rate during the Halloween GLE events}

A violent solar activity was observed in October-November 2003, which leaded to three GLEs, with onsets occurring on 28 October (Fig.1a), 29 October (Fig.1b), and on 2 November (Fig.1c), respectively. The GLE on 28 October 2003 was associated with a large flare (4B, X17.2) occurred in the active region AR10486. The GLE 65 followed significant interplanetary disturbance related to previously ejected coronal mass ejection (CME) on 26 October with correspondence with a 3B/X1.2 flare in the same active region. The GLE 66 was characterized with a smaller NM count rate increase, thus this event was weaker. A strong Forbush decrease was also observed prior and during this event (Fig.1a,b), which is explicitly considered, i.e. a GCR flux reduce is taken into account during the computations. The GLE 67 event on 2 November 2003 was related to an X8.3/2B solar flare, with onset at about 17:30-17:35 UT.

Using the model described in Section 2 and derived GLE particles spectra $[17,18]$ we computed the ion production rate in the polar and sub-polar region with rigidity cut-off $R_{c} \leq 1 \mathrm{GV}$ 
(Fig.2) and high mid-latitudes region with rigidity cut-off $R_{c} \leq 2 \mathrm{GV}$ (Fig.3). The computed over GLE 65 ion production rate was significant during the initial and main phase of the event, specifically in the polar low stratosphere (Fig. 2a). The ion production rate diminished but remained significant during the late phase of the event. The ion production rate during GLE 66 is considerably smaller, because of softer SEP spectra, the reduced SEP flux and the Forbush decrease. The ion production rate during GLE 66 is almost constant throughout the event. The ion production during the last event - GLE 67 is greater than GLE 66, specifically during the initial and main phase of the event, but rapidly diminished during the late phase. In all cases, in the region of mid latitudes with $R_{c} \leq 2 \mathrm{GV}$, the ion production rate induced by SEPs is comparable to the average due to GCR (Fig.3). Moreover, at low mid latitudes with rigidity cut-off of about $3 \mathrm{GV}$ and greater, the ion production due to GCR dominates in the whole atmosphere throughout the events.
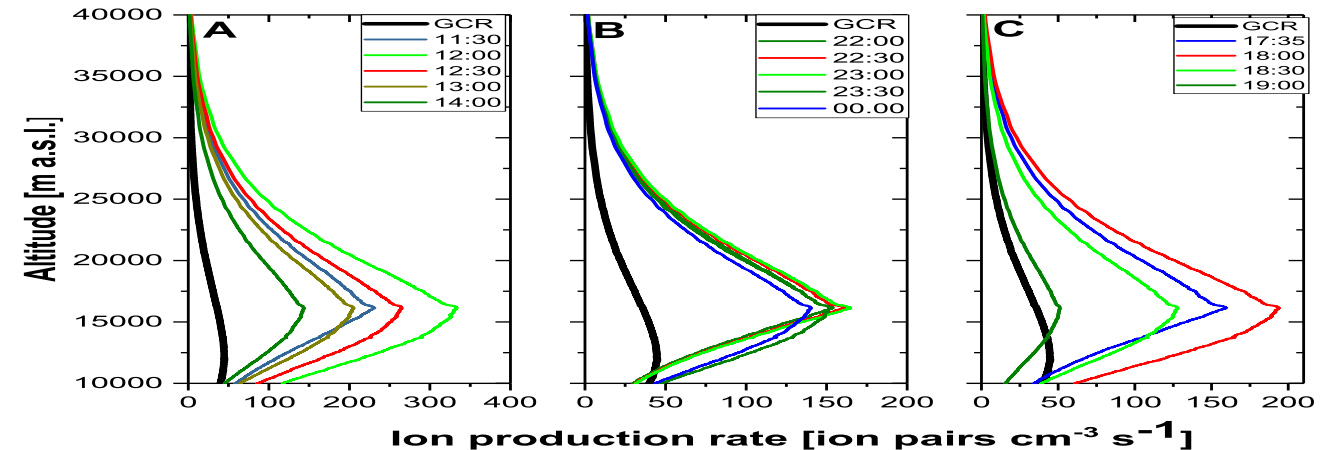

Figure 2: Ion production rate during Halloween events in the polar and sub-polar region with $R_{c} \leq$ 1 GV. a) GLE 65; b) GLE 66; c) GLE 67. The black line(s) corresponds to GCR contribution.
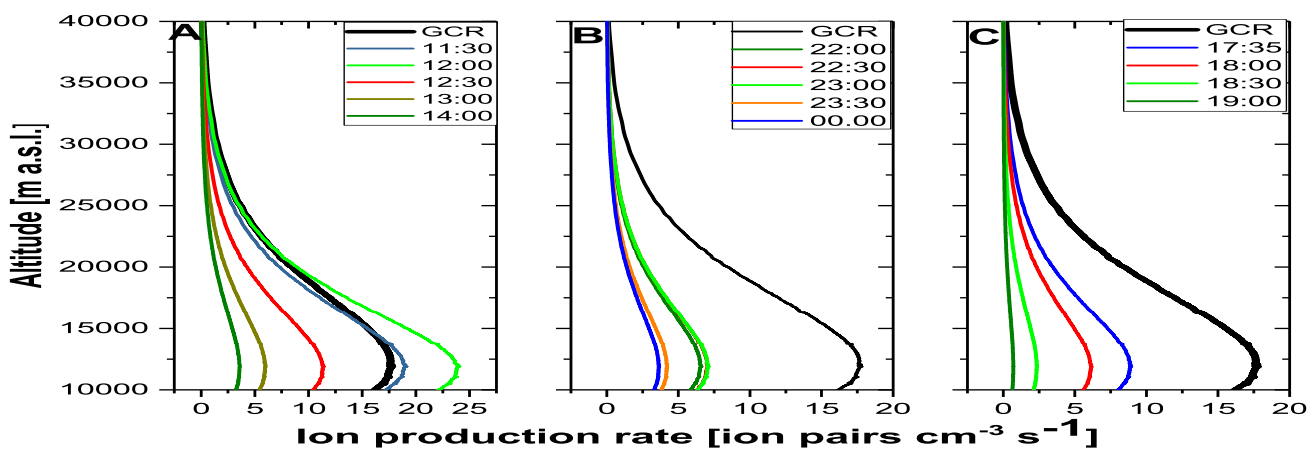

Figure 3: Ion production rate during Halloween events in mid-latitudes with $R_{c} \leq 2 \mathrm{GV}$. a) GLE 65; b) GLE 66; c) GLE 67. The black line(s) corresponds to GCR contribution.

\section{Ionization effect during the Halloween GLE events}

Using the computed ion production rates during the Halloween GLEs, presented in section 3, we computed the corresponding averaged over $24^{h}$ ionization effect similarly to [36]. The results are presented in Fig.4 for GLE 65, Fig.5 for GLE 66 and Fig.6 for GLE 67, respectively. 


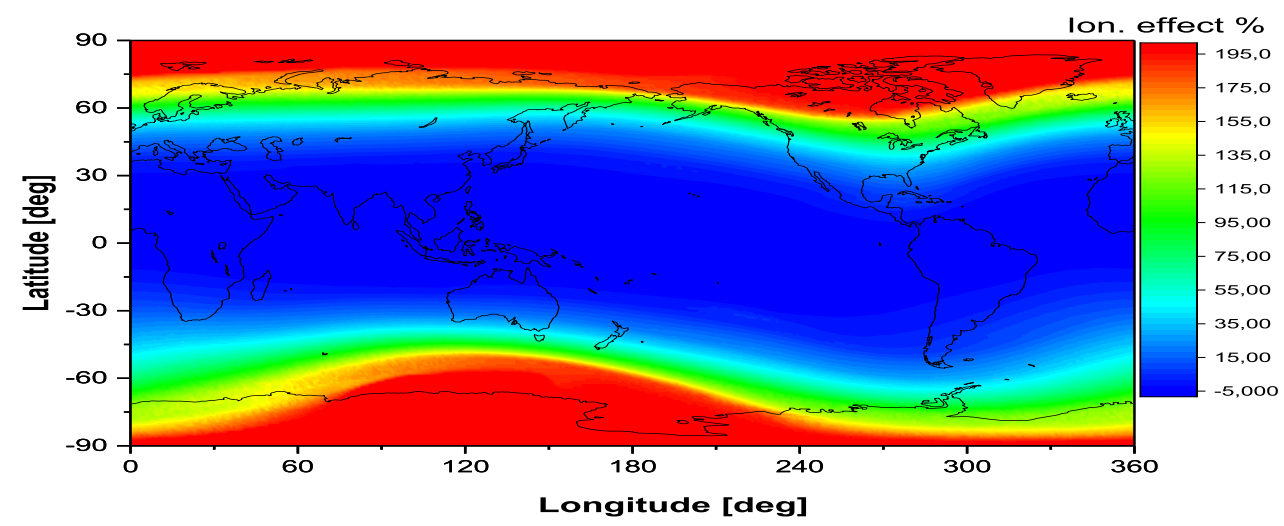

Figure 4: Global map of $24^{h}$ ionization effect in the region of the Regener-Pfotzer maximum of about $15 \mathrm{~km}$ a.s.l. during the GLE 65 on 28 October 2003.

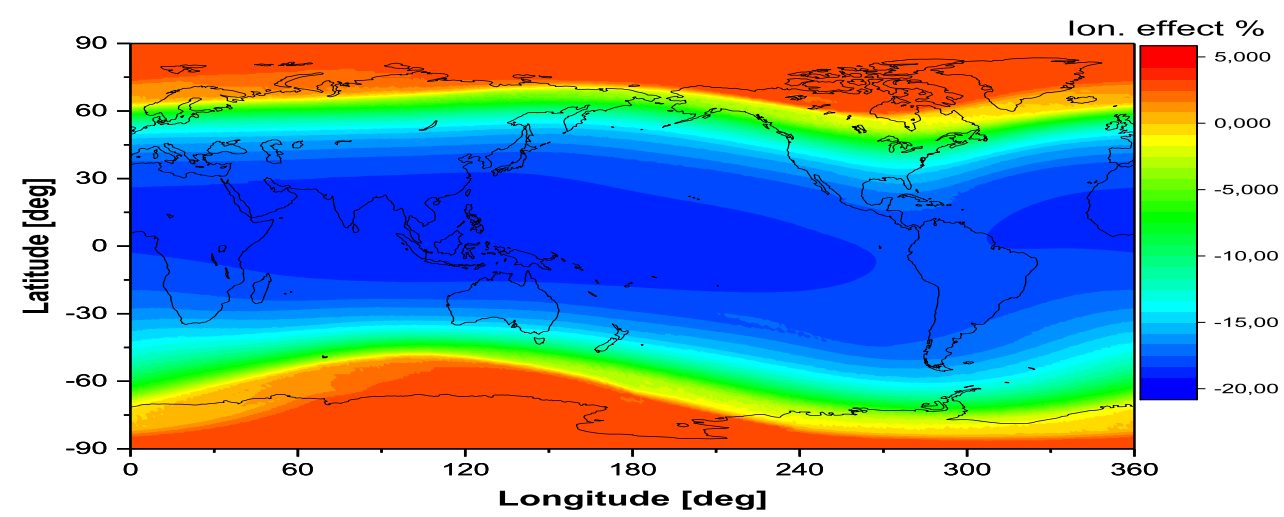

Figure 5: Global map of $24^{h}$ ionization effect in the region of the Regener-Pfotzer maximum of about $15 \mathrm{~km}$ a.s.l. during the GLE 66 on 29 October 2003.

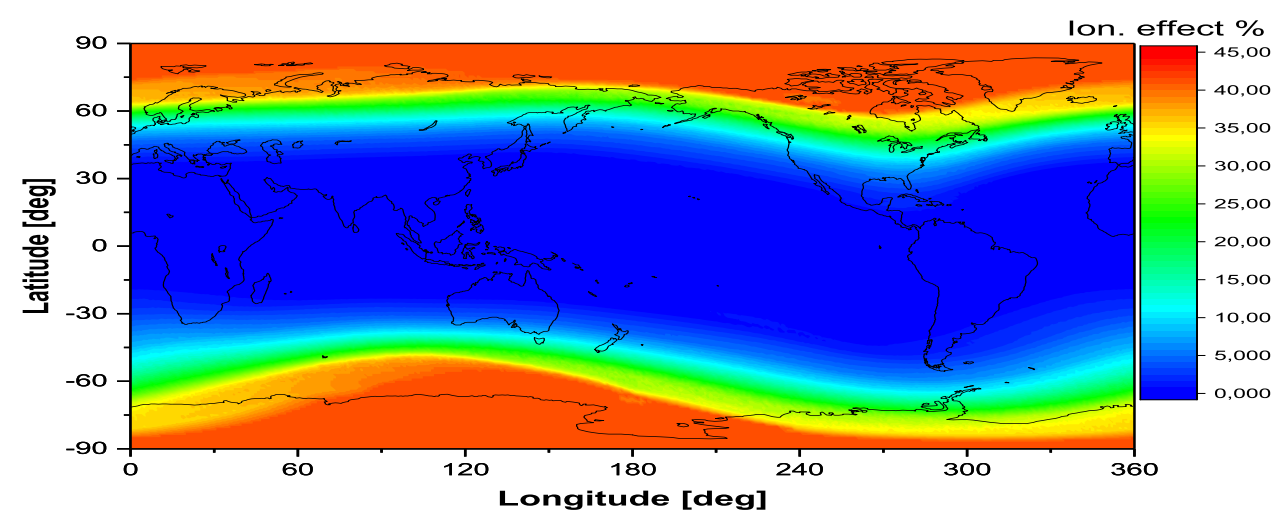

Figure 6: Global map of $24^{h}$ ionization effect in the region of the Regener-Pfotzer maximum of about $15 \mathrm{~km}$ a.s.l. during the GLE 67 on 2 November 2003. 
The ionization effect during GLE 65 is significant in the high-latitude region, but negligible in lower mid and equatorial latitudes (Fig.4). The ionization effect during GLE 66 is weak even in the polar region and is even considerably negative in the equatorial region, mostly due to reduced GCR flux (Fig.5). Accordingly, the ionization effect during GLE 67 is not significant in mid latitudes and it is marginal in the equatorial region (Fig.6).

\section{Conclusion}

Using reconstructed SEP spectra we derived the ion production rate and the corresponding ionization effect during the sequence of Halloween GLE events in October-November 2003, explicitly considering the dynamics of GCRs and GLE particles flux. It was shown that the ionization effect in the polar and sub-polar region is significant, but GLE 66 takes place in deep Forbush decrease (Fig. 1b). The ionization effect is considerably greater compared to other events during GLE 65 and GLE 67 (Fig. 1a,c). The role of the Forbush decrease is significant and considerably reduce the total i.e. the integrated over three events ionization effect, specifically in mid and low latitudes. The computed ionization effect during the Halloween events in October-November 2003 give a good basis for further studies related to the space weather and the possible impact of precipitating high-energy particles of solar origin on atmospheric chemistry and physics [37].

\section{Acknowledgements}

This work was supported by the Academy of Finland (project 307411, Center of Excellence ReSoLVE).

\section{References}

[1] I. G. Usoskin, L. Desorgher, P. Velinov, M. Storini, E. Flückiger, R. Bütikofer, and G. Kovaltsov, Ionization of the Earth's atmosphere by solar and galactic cosmic rays, Acta Geophysica 57 (2009), no. $188-101$.

[2] G. A. Bazilevskaya, I. G. Usoskin, E. Flückiger, R. Harrison, L. Desorgher, B. Bütikofer, M. Krainev, V. Makhmutov, Y. Stozhkov, A. Svirzhevskaya, N. Svirzhevsky, and G. Kovaltsov, Cosmic ray induced ion production in the atmosphere, Space Science Reviews 137 (2008) 149-173.

[3] E. Rozanov, M. Calisto, T. Egorova, T. Peter, and W. Schmutz, Influence of the precipitating energetic particles on atmospheric chemistry and climate, Surveys in Geophysics 33 (2012), no. 3-4 483-501.

[4] I. Mironova, K. Aplin, F. Arnold, G. Bazilevskaya, R. Harrison, A. Krivolutsky, K. Nicoll, E. Rozanov, E. Turunen, and I. Usoskin, Energetic particle influence on the Earth's atmosphere, Space Science Reviews (2015) 96.

[5] E. Regener and G. Pfotzer, Vertical intensity of cosmic rays by threefold coincidences in the stratosphere, Nature 136 (1935) 718-719.

[6] G. Pfotzer, Dreifachkoinzidenzen der ultrastrahlung aus vertikaler richtung in der stratosphäre, Zeitschrift für Physik 102 (1936), no. 1-2 23-40.

[7] P. Stoker, L. Dorman, and J. Clem, Neutron monitor design improvements, Space Science Reviews 93 (2000), no. 1-2 361-380. 
[8] S. Poluianov, I. Usoskin, A. Mishev, A. Shea, and D. Smart, GLE and sub-GLE redefinition in the light of high-altitude polar neutron monitors, Solar Physics 292 (2017), no. 11176.

[9] A. Mishev, P. Velinov, L. Mateev, and Y. Tassev, Ionization effect of solar protons in the Earth atmosphere - case study of the 20 January 2005 SEP event, Advances of Space Research 48 (2011), no. 7 1232-1237.

[10] I. Usoskin, G. Kovaltsov, I. Mironova, A. Tylka, and W. Dietrich, Ionization effect of solar particle GLE events in low and middle atmosphere, Atmospheric Chemistry and Physics 11 (2011) 1979-1988.

[11] A. Mishev, P. Velinov, L. Mateev, and Y. Tassev, Ionization effect of nuclei with solar and galactic origin in the earth atmosphere during GLE 69 on 20 January 2005, Journal of Atmospheric and Solar-Terrestrial Physics 89 (2013), no. 1 1-7.

[12] I. Mironova, I. Usoskin, G. Kovaltsov, and S. Petelina, Possible effect of extreme solar energetic particle event of 20 January 2005 on polar stratospheric aerosols: Direct observational evidence, Atmospheric Chemistry and Physics 12 (2012), no. 2 769-778.

[13] I. Usoskin, O. Gladysheva, and G. Kovaltsov, Cosmic ray-induced ionization in the atmosphere: Spatial and temporal changes, Journal of Atmospheric and Solar-Terrestrial Physics 66 (2004), no. 18 1791-1796.

[14] L. Desorgher, E. Flückiger, M. Gurtner, M. Moser, and R. Bütikofer, A GEANT 4 code for computing the interaction of cosmic rays with the earth's atmosphere, International Journal of Modern Physics A 20 (2005), no. A11 6802-6804.

[15] I. Usoskin and G. Kovaltsov, Cosmic ray induced ionization in the atmosphere: Full modeling and practical applications, Journal of Geophysical Research 111 (2006), D21206.

[16] P. Velinov, A. Mishev, and L. Mateev, Model for induced ionization by galactic cosmic rays in the earth atmosphere and ionosphere, Advances in Space Research 44 (2009), no. 9 1002-1007.

[17] L. Miroshnichenko, K.-L. Klein, G. Trottet, P. Lantos, E. Vashenyuk, Y. Balabin, and B. Gvozdevsky, Relativistic nucleon and electron production in the 2003 October 28 solar event, Journal of Geophysical Research: Space Physics 110 (2005), no. A9 A09S08.

[18] L. Kocharov, S. Pohjolainen, A. Mishev, M. Reiner, J. Lee, T. Laitinen, L. Didkovsky, V. Pizzo, R. Kim, A. Klassen, M. Karlicky, K.-S. Cho, D. Gary, I. Usoskin, E. Valtonen, and R. Vainio, Investigating the origins of two extreme solar particle events: Proton source profile and associated electromagnetic emissions, Astrophysical Journal 839 (2017), no. 279.

[19] A. Mishev and P. Velinov, Atmosphere ionization due to cosmic ray protons estimated with CORSIKA code simulations, Comptes Rendus de l'Académie Bulgare des Sciences 60 (2007), no. 3225-230.

[20] H. Porter, C. Jackman, and A. Green, Efficiencies for production of atomic nitrogen and oxygen by relativistic proton impact in air, The Journal of Chemical Physics 65 (1976), no. 1 154-167.

[21] A. Mishev and P. Velinov, Contribution of cosmic ray nuclei of solar and galactic origin to atmospheric ionization during sep event on 20 january 2005, Comptes Rendus de l'Académie Bulgare des Sciences 65 (2012), no. 3 373-380.

[22] L. Gleeson and W. Axford, Solar modulation of galactic cosmic rays, Astrophysical Journal 154 (1968) 1011-1026.

[23] R. Burger, M. Potgieter, and B. Heber, Rigidity dependence of cosmic ray proton latitudinal gradients measured by the Ulysses spacecraft: Implication for the diffusion tensor, Journal of Geophysical Research 105 (2000) 27447-27445. 
[24] I. Usoskin, K. Alanko-Huotari, G. Kovaltsov, and K. Mursula, Heliospheric modulation of cosmic rays: Monthly reconstruction for 1951-2004, Journal of Geophysical Research 110 (2005), A12108.

[25] A. Mishev and I. Usoskin, Analysis of the ground level enhancements on 14 July 2000 and on 13 December 2006 using neutron monitor data, Solar Physics 291 (2016), no. 4 1225-1239.

[26] A. Mishev, S. Poluianov, and I. Usoskin, Assessment of spectral and angular characteristics of sub-GLE events using the global neutron monitor network, Journal of Space Weather and Space Climate 7 (2017) A28.

[27] A. Mishev, I. Usoskin, O. Raukunen, M. Paassilta, E. Valtonen, L. Kocharov, and R. Vainio, First analysis of GLE 72 event on 10 September 2017: Spectral and anisotropy characteristics, Solar Physics 293 (2018) 136.

[28] J. Picone, A. Hedin, D. Drob, and A. Aikin, NRLMSISE-00 empirical model of the atmosphere: Statistical comparisons and scientific issues, Journal of Geophysical Research: Space Physics $\mathbf{1 0 7}$ (2002), no. A12 1468.

[29] A. Mishev and P. Velinov, Influence of hadron and atmospheric models on computation of cosmic ray ionization in the atmosphere-extension to heavy nuclei, Journal of Atmospheric and Solar-Terrestrial Physics 120 (2014) 111-120.

[30] A. Mishev and P. Velinov, A maverick GLE 70 in solar minimum. calculations of enhanced ionization in the atmosphere due to relativistic solar energetic particles, Comptes Rendus de l'Académie Bulgare des Sciences 66 (2013), no. 10 1457-1462.

[31] A. Mishev and P. Velinov, Time evolution of ionization effect due to cosmic rays in terrestrial atmosphere during GLE 70, Journal of Atmospheric and Solar-Terrestrial Physics 129 (2015) 78-86.

[32] A. Mishev and P. Velinov, Ionzation rate profiles due to solar and galactic cosmic rays during GLE 59 on Bastille day 14 July 2000, Comptes Rendus de l'Académie Bulgare des Sciences 68 (2015), no. 3 359-366.

[33] A. Mishev and P. Velinov, Determination of medium time scale ionization effects at various altitudes in the stratosphere and troposphere during ground level enhancement due to solar cosmic rays on 13.12.2006 (GLE 70), Comptes Rendus de l'Académie Bulgare des Sciences 68 (2015), no. 11 $1425-1430$.

[34] A. Mishev and P. Velinov, Ionization effect due to cosmic rays during Bastille day event - GLE 59 on short and mid time scales, Comptes Rendus de l'Académie Bulgare des Sciences 69 (2016), no. 11 1479-1484.

[35] A. Mishev and P. Velinov, Long term ionization effect during several GLE events of solar cycle 23 comparative analysis, Proceedings of Science (2017) 135186.

[36] A. Mishev and P. Velinov, Ion production and ionization effect in the atmosphere during the Bastille day GLE 59 due to high energy SEPs, Advances in Space Research 61 (2018), no. 1 316-325.

[37] Y. Tassev, N. Kilifarska and D. Tomova, Statistical analysis of solar proton flux influence on thermodynamics of middle atmosphere in the North hemisphere, Comptes Rendus de l'Académie Bulgare des Sciences 67 (2014), no. 1 95-100. 